\title{
A New Model for Fast Analysis of Leveling Process Petruska Jindrich $^{1, a}$, Navrat Tomas ${ }^{1, b}$ and Sebek Frantisek ${ }^{1, c}$ \\ ${ }^{1}$ Brno University of Technology, Faculty of Mech.Engineering Technicka 2, 61669 Brno, Czech Republic \\ apetruska@fme.vutbr.cz, bnavrat@ fme.vutbr.cz, c y107598@stud.fme.vutbr.cz
}

Keywords: Leveling Process, FEM, plastic bending.

\begin{abstract}
The paper deals with numerical analysis of the process of roller leveling of long products. The problem of multiple elastic plastic bending is solved by a program in Matlab, which is based on the Finite Element Method. The aim is to provide a simple, user friendly tool, capable of quick and reliable analysis of the process without the necessity to work with large multipurpose FEM packages. Direct solution of the problem is formulated, starting from the roll intermeshing and product geometry. Roller loading, product deflection, curvature and plastification are the output parameters of the program. With fast and reliable solution of the direct problem, optimal setting of the leveling process will be sought in an iterative process.
\end{abstract}

\section{Introduction}

Principal idea of all types of leveling processes is based on repeated elastic plastic bending, which results in redistribution of residual stress with the aim of minimization of final curvature of the product. It is reported that this process is highly parameter sensitive and effective analysis of the process is necessary to be able to optimize the setting of the leveling machine with respect to measured input parameters. Principal role is played by the relation between the intermeshing of leveling rollers and appropriate product curvature distribution, which leads to optimal distribution of residual stress and minimal output curvature. This problem is complicated due to its inherent nonlinearity, dependence on loading history, instability and sensitivity to material cyclic plasticity characteristics [1], [2].

Historically, effective models to solve the above problem were based on empirical experience combined with elementary qualitative analytical models [3], [4]. With the development of FEM and increased computer power which enables to solve nonlinear problems effectively, more complicated models were formulated and many phenomena of the leveling process could be investigated computationally. However, it is still difficult to use this method in engineering practice due to its capacity and time demands [5], [6]. That is the reason, why a number of simpler computational models for quick and simple analysis of the leveling process were formulated. Generally, they are based on the integration of curvature of the leveled product, starting from the bending curvature on each roller. Many versions of this approach were published in previous years [7]-[9].

In our paper we suggest an inverse approach, starting from the roller intermeshing. Fast algorithm is used then to evaluate all necessary variables like curvatures, bending moments and roller loadings. Full stress-strain history is taken into account and residual stress distribution with final curvature can be easily obtained. The algorithm can be modified to different material models by changing appropriate subroutine, it can be also used for different types of leveling processes. It can be a basis for leveling optimization procedure. In the paper we shall present our experience with stability and general effectiveness of the suggested algorithm on the example of long products leveling. 


\section{Numerical modelling of the leveling process}

In Fig. 1 we show schematic representation of the 7-roller leveling machine. We suppose long products with simple bending theory of beams and one dimensional state of stress. Material is moving through the machine from the left side, bottom rollers are vertically fixed, whereas the four top rollers are adjustable to obtain optimal bending to straighten the product.

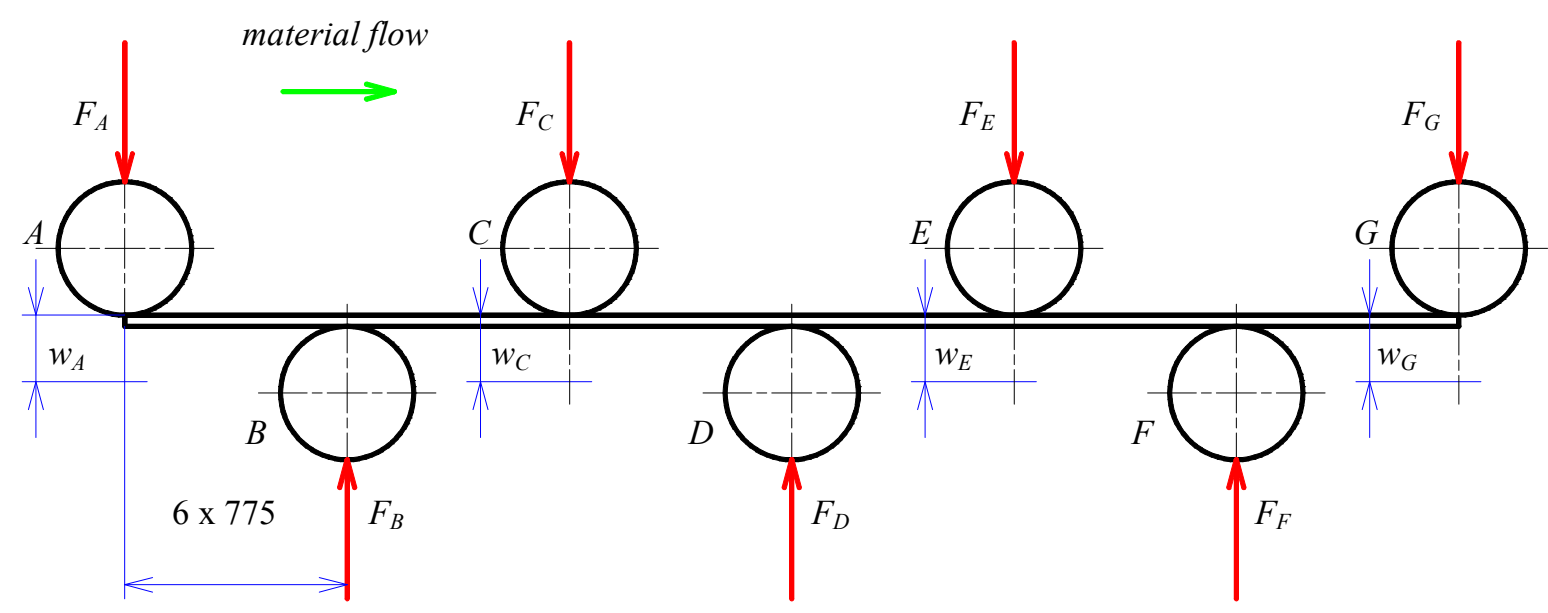

Fig. 1: Schematic representation of the leveling process

We also suppose simple point contact between the rollers and the material. Geometrical input parameters are represented by the beam cross section, input curvature and by positions of the adjustable rollers. Material model is characterised by bilinear flow curve with linear elastic and linear hardening diagram, von Mises criterion of plasticity and kinematic hardening rule. Output parameters are the deflection, slope and curvature along the beam, distribution of bending moments, shear forces and roller reactions and distribution of longitunidal elastic plastic stress in any cross section along the beam, including residual stress and curvature at the end (Fig.3).

Basic equations and solution algorithm. The algorithm is based on iterative solution of a linearised equation

$$
\begin{aligned}
& \mathbf{K}_{T, i-1} \cdot \Delta \mathbf{U}_{i}=\mathbf{R}_{i-1}, \\
& \mathbf{U}_{i}=\Delta \mathbf{U}_{i}+\mathbf{U}_{i-1},
\end{aligned}
$$

where $\mathbf{K}_{\mathrm{T}}$ is tangential stiffness matrix composed of 2-node FE beam elements with two degrees of freedom in each node (displacement and slope - see [10]) and $\mathbf{R}$ is a matrix of residual nodal shear forces and bending moments.

Correction for nonlinear material behaviour is realized each iteration. It is based on the computation of curvature in each node, from which the total, elastic and plastic strain can be easily obtained together with the distribution of longitudinal elastic plastic strain across the beam cross section (Fig.2). To take into account the flow of material through the leveling machine, Euler-type scheme is adopted in tracking the history of plastic deformation. Development of the plastic deformation linked with the history of stress distribution is always tracked from the first node on the left side of the beam with curvature increments corresponding to subsequent nodes of the beam, until the last node is reached. The state in the last node for converged solution then corresponds to residual stress and curvature of the beam leaving the leveling machine.

Nonlinear distribution of stress according to Fig. 2 results in correction of bending moment, which is modified according to

$$
M_{m}=\iint \sigma(z) \cdot z d S
$$


where $z$ is the vertical coordinate and $S$ the cross section area. From the modified moment, equivalent nodal loading matrix $\mathbf{f}$ is easily computed for each element

$$
\mathbf{f}=\int_{0}^{L} \mathbf{B}(x) \cdot M_{m}(x) \cdot \mathrm{d} x,
$$

where $\mathrm{B}(\mathrm{x})$ is the curvature approximation matrix according to [10], $L$ is the element length and $M_{m}(x)$ is a linear approximation of the modified bending moment along the finite element, obtained from the values in nodal points. Global matrix of equivalent nodal loads $\mathbf{F}_{\mathrm{i}}$ is then obtained by contributions from all finite elements and matrix of residual loads for the next iteration is $\mathbf{R}_{\mathrm{i}}=-\mathbf{F}_{\mathrm{i}}$.

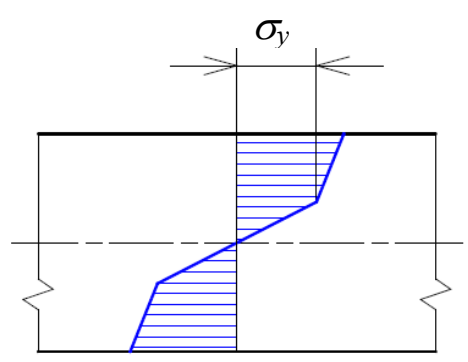

Fig. 2: Distribution of elastic plastic bending stress $\sigma$

In case of growing plastic strain, i.e. for active plastic loading, also the stiffness matrix must be modified. It is realized by changing the beam flexural rigidity according to

$$
\begin{aligned}
& (E J)_{m}=\iint E_{m}(z) \cdot z^{2} d S \quad, \quad \text { where } \\
& E_{m}=E \cdot\left(1-\frac{E}{E+H}\right) \text { and } H=\frac{E \cdot E_{T}}{E-E_{T}} .
\end{aligned}
$$

$E, E_{T}$ is the modulus of elasticity and tangential hardening modulus, respectively - see [11]. Modified flexural rigidity according to Eq.(5) enters the stiffness matrix of each plastified element. It results in a new tangential stiffness matrix $\mathbf{K}_{\text {Ti }}$ prepared for the next iteration.

Finally, the convergence criteria are checked and the procedure is either stopped or returned back to Eq.1 with iteration number increased by one.

\section{Testing results}

Performance of the developed program is illustrated by the following testing example: Circular cross section bar with diameter of $70 \mathrm{~mm}$ is moved through the leveling machine with subsequent intermeshing of adjustable rollers: 4, 12, 10 and $2 \mathrm{~mm}$. Material is characterized by yield stress of 350 $\mathrm{MPa}$ and no hardening. In Fig. 3 we show the deflection, slope, curvature, bending moment and maximal bending stress along the length of the leveled bar. The diagram of curvature shows typical nonlinear stages in plastic bending sections in comparison to linear bending moment diagram. The last diagram shows the distribution of residual stress over the cross section of the bar after leaving the machine. Loading of leveling rollers A-G (Fig.1) is $-18.1 \mathrm{kN}, 59.3 \mathrm{kN},-88.6 \mathrm{kN}, 96.3 \mathrm{kN},-95.1 \mathrm{kN}$, $67.9 \mathrm{kN}$ and $-21.7 \mathrm{kN}$.

The results correspond to detailed FEM analysis realized by Ansys, the solution procedure is stable and very fast in comparison to multipurpose FEM package. This performance, together with user friendly interface makes our program an ideal tool for optimization of the leveling process. More results and discussion will be presented at the conference. 


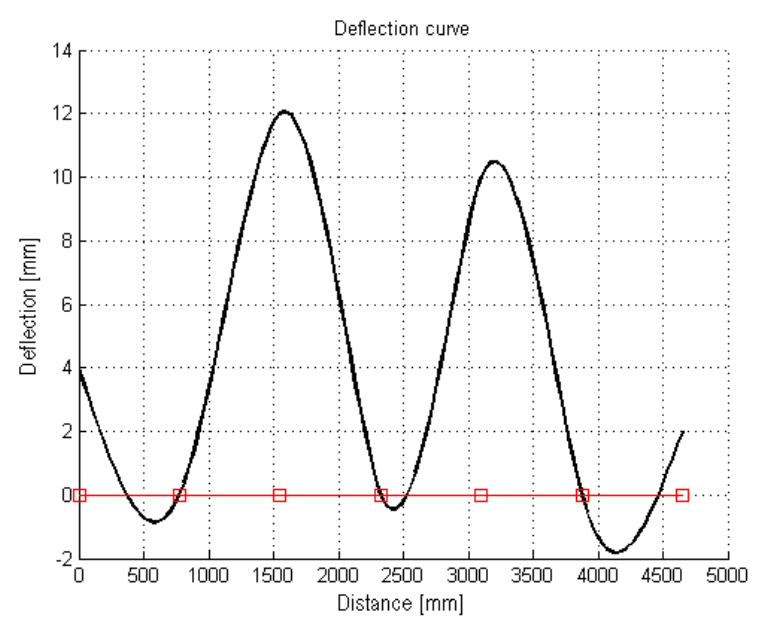

Fig 3a) Deflection [mm]

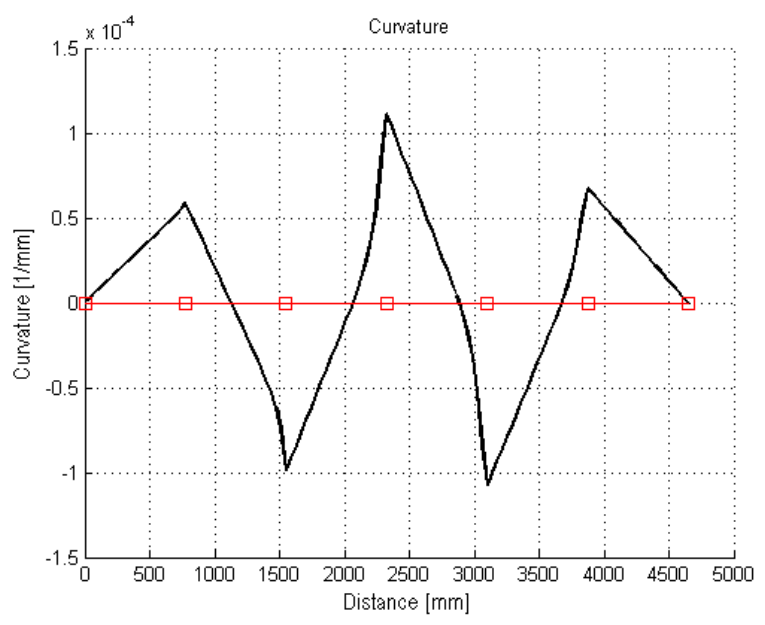

Fig 3c) Curvature $\left[\mathrm{mm}^{-1}\right]$

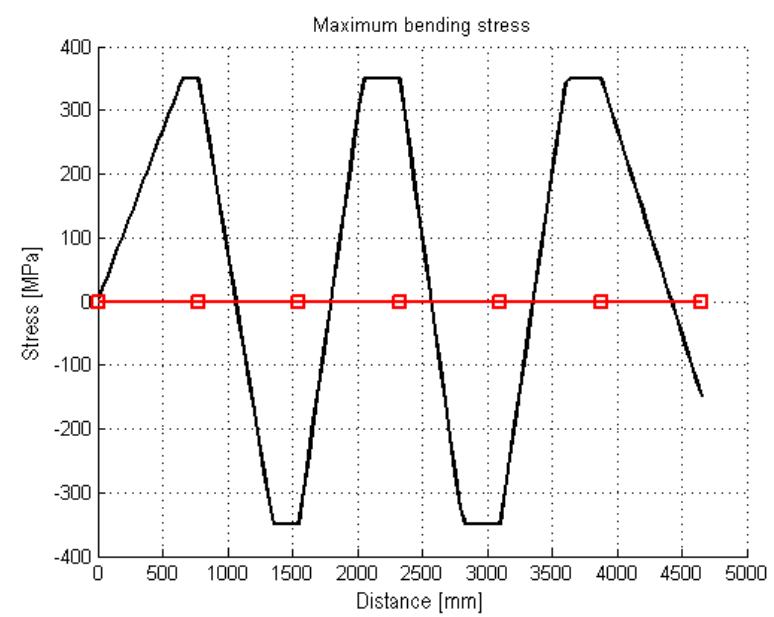

Fig 3e) Max. bending stress [MPa]

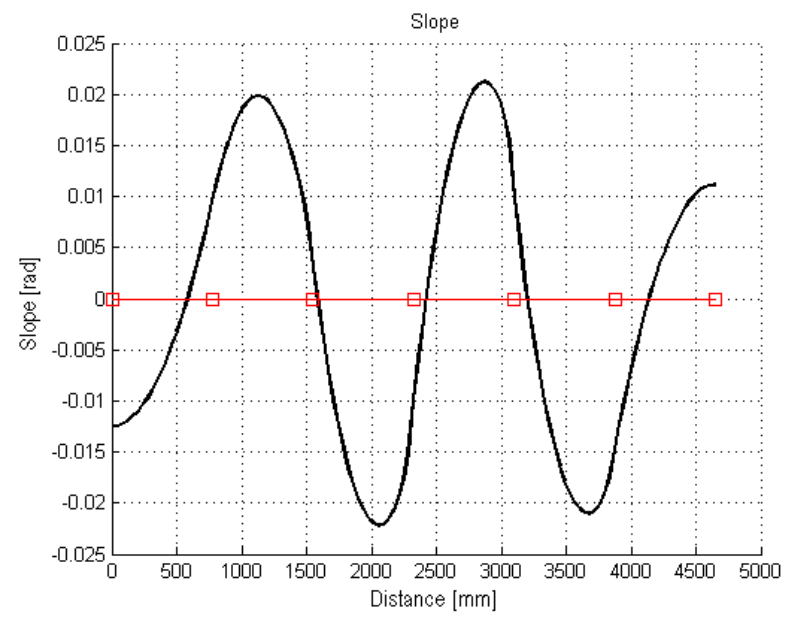

Fig 3b) Slope [rad]

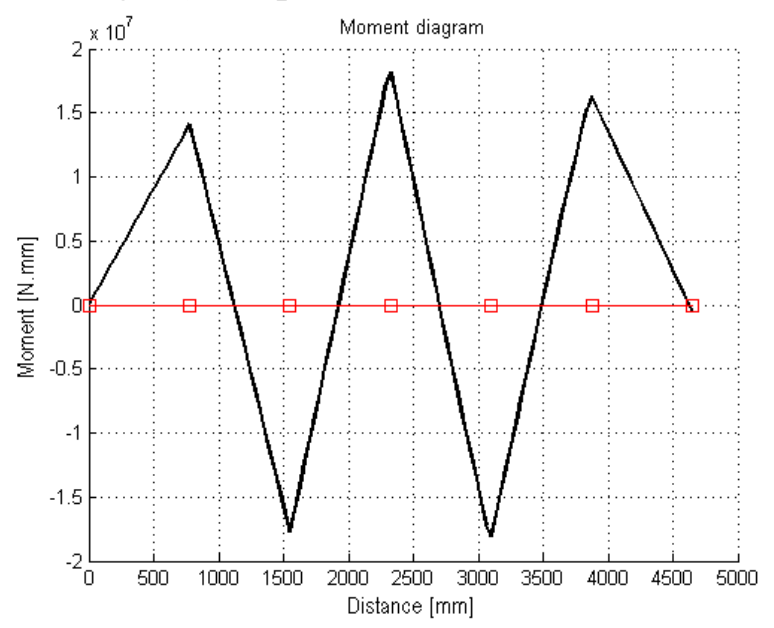

Fig 3d) Bending moment [Nmm]

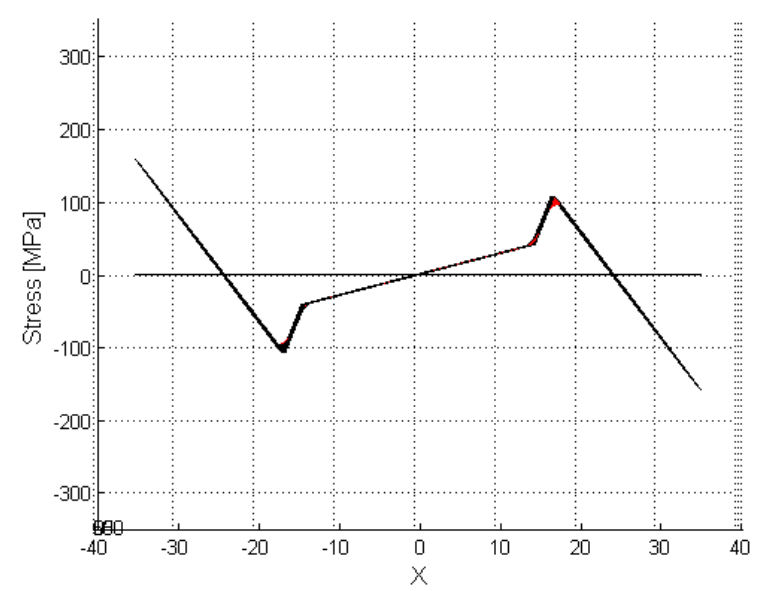

Fig 3f) Residual stress [MPa]

Fig. 3: Results of the testing example: deflection, slope, curvature, bending moment and bending stress along the leveled bar, residual stress across the bar section

\section{Summary}

User friendly and fast program for direct solution of leveling process of long products was developed and tested. Its modular structure is prepared to enhance its applicability to other similar processes like plate leveling, tensile leveling of thin sheets, cross roll straightening of bars and tubes, etc. Some improvements are being prepared also in precise evaluation of position of the contact point between 
the rollers and product. Other improvements should include the influence of shear force to product deflection or better modelling of material. Especially the cyclic hardening/softening of material can have a substantial influence on the results.

Realized testing proved that the algorithm provides fast and reliable results and can be used as an ideal tool for optimization of many types of leveling processes. This will be the main direction of further development with strong accent to practical industrial applicability of the results.

\section{Acknowledgement}

Financial help of the grant projects GA101/09/1630 and European Regional Development Fund project CZ.1.05/2.1.00/01.0002 is gratefully acknowledged.

\section{References}

[1] M. Nastran, K. Kuzman: J. Mat. Proc. Tech. 133 (2003) 166-173.

[2] A. Mutrux, B. Berisha, P. Hora: J. Mat. Proc. Tech. 211 (2011) 1448-1456.

[3] H.Tokunaga: Bul. JSME 15 (1961) 605-611.

[4] Z. Marciniak: Theory of sheet forming (in Polish, PWN Warszaw, 1961).

[5] H. Huh, J.H. Heo, H.W. Lee: Int. J. Mach. Tool. Manuf. 43 (2003) 345-350.

[6] B. C. Biempica et. al.: Appl. Math. Model. 33 (2009) 34-53.

[7] E. Doege, R. Menz, S. Huinink: Manuf. Technol. 51(2002) 191-194.

[8] Z. Liu, Y. Wang, X.Yan: Int. J. Mech. Sci. 54 (2012) 213-224.

[9] M. Nastran, K. Kuzman: J. Mat. Proc. Tech. 125-126 (2002) 711-719

[10] R.D.Cook: Concepts and Applications of Finite Element Analysis, J.Wiley, 1981

[11] D.R.J. Owen, E.Hinton: Finite Elements in Plasticity, Pineridge Press, Swansea, 1980 